\title{
The Concept of Autonomy
}

\author{
Tim McFarland
}

This chapter investigates the notion of autonomy as it applies to software and cyberphysical systems, with a focus on matters which bear some significance to the application of relevant bodies of international law. As autonomy, in the context of software, is a technical term rather than a legal or philosophical one, a bottom-up approach is taken, beginning with a description of the technical origins and meaning of the term 'autonomous'. Based on that, two important relationships are discussed: that between the autonomous system and its environment, and between the system and its operator. Finally, several aspects of autonomy of relevance to a legal analysis are discussed: autonomous systems do not necessarily behave differently to manually operated systems; autonomous systems are not 'independent' of humans for the purposes of a legal analysis; and the relative contributions of human and machine in an operation involving an autonomous system are likely to be complex and variable, such that they should arguably be viewed as a form of collaboration between human and machine rather than a simple delegation of the entirety of a task to a machine.

Dr Tim McFarland is a Research Fellow in the University of Queensland Law School's Law and the Future of War Research Group [t.mcfarland@uq.edu.au].

This research was funded by the Air Force Office of Scientific Research (award number FA9550-18-1-0181). Any opinions, finding, and conclusions or recommendations expressed in this material are those of the author and do not necessarily reflect the views of the United States Air Force.

A revised version of this paper will appear as a chapter in Rain Liivoja and Ann Väljataga (eds), Autonomous Cyber Capabilities under International Law (Tallinn: NATO CCDCOE, forthcoming in 2021). 


\section{Contents}

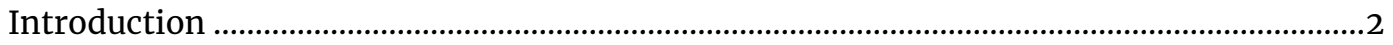

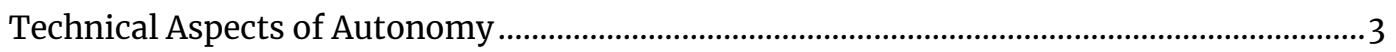

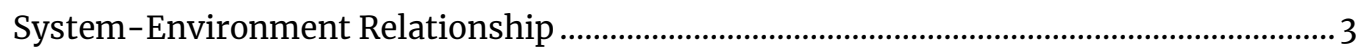

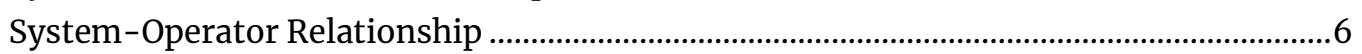

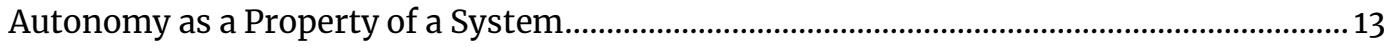

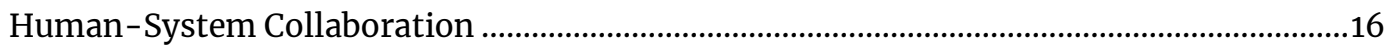

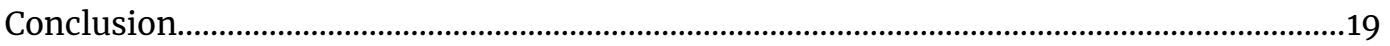

\section{Introduction}

This chapter discusses the notion of autonomy as it applies to software and cyber-physical systems. The purpose is to identify and explain those aspects of autonomous cyber capabilities which bear some significance to the application of relevant bodies of international law. In that respect, the chapter expands upon the outline of various conceptions of autonomy presented in a working paper produced by the NATO Cooperative Cyber Defence Centre of Excellence. ${ }^{1}$

In the debate about regulating the development and use of autonomous systems, much confusion has resulted from different commentators adopting different definitions of autonomy, either explicitly or implicitly, with the results of their analyses varying considerably depending on the definition adopted. Rather than risk adding to that confusion, the analysis herein takes a more direct approach. The first substantive section below presents a study of the efforts by developers of software and cyber-physical systems to impart certain capabilities to those systems, including an overview of the capabilities being sought and the technologies being employed in their pursuit. Based on that material, the second section defines and discusses two legally relevant properties of autonomous software systems. The third and final section discusses the ways in which the roles of human and software system may vary in operations involving autonomous software.

The discussion is framed largely in abstract terms. It is not a detailed case study of a specific program, software development technology or device, although numerous examples are employed where appropriate. Rather, it addresses an abstract phenomenon, being the capacity of a software application or a cyberphysical system to operate autonomously, however that capacity may be achieved and whatever the physical form (or lack thereof) of the system involved. Indeed, much of this discussion is as applicable to a study of autonomous robots as it is to a study of software systems: a software-based control system built into a robot

\footnotetext{
${ }^{1}$ Rain Liivoja, Maarja Naagel and Ann Väljataga, 'Autonomous Cyber Capabilities under International Law' (2019) NATO Cooperative Cyber Defence Centre of Excellence Working Paper, 7-11 $<$ https://ccdcoe.org/library/publications/autonomous-cyber-capabilities-under-internationallaw $>$ accessed 20 January 2020.
} 
and constrained to interact with the outside world through the hardware components of that robot can do so autonomously just as a software-only autonomous system can interact directly with its environment. Accordingly, frequent reference is made to autonomous 'systems', rather than specifically to software, robots, machines, weapons, or other devices. An abstract approach is useful, even necessary, given that the specific technologies which enable autonomy in artificial systems are developing rapidly. It is desirable that the findings presented here, and any legal reasoning based on them, remain useful as the underlying technologies progress.

\section{Technical Aspects of Autonomy}

'Autonomous', in the context of cyber capabilities, is not a term selected by lawyers or philosophers; it was selected by scientists and engineers to describe a desired outcome of their work on software and hardware systems. It is a property of a technological system, a degree of which has been achieved in some systems in use today and greater degrees of which are the goal of research and development programs in relevant fields. This investigation therefore begins with a brief study of the development outcome which the term 'autonomous' was selected to describe.

The essence of autonomy in a software context is, as in other contexts, selfregulation or self-governance. It is a concept which may be viewed in two ways: that the system in question generates the rules by which it operates in its environment, and that no other entity generates the rules by which the system operates. Those two sides of the autonomy coin are equally important and equally worthy of further discussion, given the confusion they have caused at various stages of the debate about regulation of autonomous military systems. They are discussed below in terms of two relationships: that between the system and its task or environment, and that between the system and its operator.

\section{System-Environment Relationship}

Abbass, Scholz and Reid provide a useful conceptualisation of autonomy in a technical context:

Foundationally, autonomy is concerned with an agent that acts in an environment. However, this definition is insufficient for autonomy as it requires persistence (or resilience) to the hardships that the environment acts upon the agent. An agent whose first action ends in its demise would not demonstrate autonomy. The themes of autonomy then include agency, persistence and action...Action may be 
understood as the utilisation of capability to achieve intent, given awareness. $^{2}$

Likewise, Franklin and Graesser explain that

An autonomous agent is a system situated within and a part of an environment that senses that environment and acts on it, over time, in pursuit of its own agenda and so as to effect what it senses in the future. ${ }^{3}$

('Agent', in software terms, refers to a software entity which acts autonomously in an environment in pursuit of some goal for which it was designed. ${ }^{4}$ )

In other words, a software entity is autonomous when it possesses both an 'intent' (an encoded representation of a goal, a purpose, or a task to be completed) and the ability to act within its environment in furtherance of that goal. Acting within an environment in furtherance of a goal in turn requires 'awareness' (the ability to sense the environment and changes therein), 'capability' (a facility for effecting desirable changes in the environment and resisting or correcting undesirable changes) and, implicitly, a means to select the capability which best serves the software's purpose in response to a given change in the environment (where that selection process may be characterised as reasoning, choice, decision-making, and so on). Overall, autonomous behaviour may be viewed as the process of aligning the software entity's awareness with its goal:

If 'capability' is defined as anything that changes the agent's awareness of the world (usually by changing the world), then the error between the agent's awareness and intent drives capability choice in order to reduce that error. Or, expressed compactly, an agent seeks achievable intent. ${ }^{5}$

Notably, that conceptualisation of autonomy lacks sharply defined thresholds, and so invites consideration of whether all software which is created for a purpose and which can detect and respond to changes in its environment may be considered to be autonomous. On the one hand, the behaviours described above (pursuing goals, sensing and effecting changes in an environment) are quite obviously matters of degree, abilities which may be exhibited in different measure by different systems in different environments. On the other hand, many

\footnotetext{
${ }^{2}$ Hussein A Abbass, Jason Scholz and Darryn J Reid, 'Foundations of Trusted Autonomy: An Introduction' in Hussein A Abbass, Jason Scholz and Darryn J Reid (eds), Foundations of Trusted Autonomy (Springer 2018) 1.

${ }^{3}$ Stan Franklin and Art Graesser, 'Is It an Agent, or Just a Program? A Taxonomy for Autonomous Agents' in Jörg P Müller, Michael J Wooldridge and Nicholas R Jennings (eds), Intelligent Agents III: Agent Theories, Architectures, and Languages (Springer 1997) 25.

4 ibid 24.

${ }^{5}$ Abbass, Scholz and Reid (n 2) 1.
} 
authors take the view that autonomous systems are a rigidly defined class of system which may be distinguished from non-autonomous or merely automated systems on the basis of objective criteria.

The notion of a device which can automatically respond to changes in its environment in order to fulfil its purpose is much older than software or robots. James Watt's centrifugal governor for steam engines, dating to approximately 1788 , is often cited as an example. Likewise, a thermostat, whether implemented in software or hardware, exhibits the essence of this behaviour. Indeed, any device which employs a closed loop control system of some sort might arguably qualify.

However, the use of 'autonomous' as a description of a self-governing device is much more recent and, in practice, the term is not generally used in reference to simple devices like governors and thermostats. Its use came about with efforts to develop systems which can perform their tasks unattended in increasingly complex circumstances (whether that complexity be in the task or the environment). While there is no precise threshold, the term is generally associated with self-governing machines whose task requires higher levels of 'algorithmic and hardware sophistication' ${ }^{6}$ and the ability to operate in the face of uncertainty:

Autonomy is more or less understood as a requirement for operating in complex environments that manifest uncertainty; without uncertainty relatively straightforward automation will do, and indeed the autonomy is generally seen here as being predicated on some form of environmental uncertainty. ${ }^{7}$

Thus, in terms that are perhaps more fitting for legal purposes, a self-governing system is more likely to be described as 'autonomous' where human observers lack the ability to precisely foresee the exact sequence of steps that the system must take in order to complete its assigned task (or, equivalently, cannot foresee all events that will transpire when the system is activated). The term is used when the high level goal of deploying the system is defined in advance but not necessarily every low level step that will be completed in its pursuit. That is, some reliance is placed upon an autonomous system to select the appropriate response to changes in its environment from among the possible responses supported by its capabilities.

\footnotetext{
${ }^{6}$ Darryn J Reid, 'An Autonomy Interrogative' in Hussein A Abbass, Jason Scholz and Darryn J Reid (eds), Foundations of Trusted Autonomy (Springer 2018) 365.

${ }^{7}$ Abbass, Scholz and Reid (n 2) 7.
} 
Some advanced software systems being developed today, known by the recently coined term 'cyber reasoning systems' ${ }^{8}$ (CRS) demonstrate this quality. Referring primarily to the systems such as those which have been deployed in DARPA's 'Cyber Grand Challenge', CRS 'combine various tools, techniques and expert knowledge to create fully autonomous systems that perform automated vulnerability detection, exploit generation and software patching in binary software without human intervention.' 9 They comprise multiple sub-systems with both offensive and defensive roles to search for vulnerabilities in adversaries' systems, develop tools for exploiting those vulnerabilities and conduct attacks against them while simultaneously searching for and repairing vulnerabilities in friendly systems under their protection and intercepting attacks against those systems launched by adversaries.

Deployed in competition with other CRS (or, hypothetically, against any intelligent adversary), human operators could not intervene to manage the use of each of those capabilities; the reasoning required for that purpose had to be encoded into each CRS such that it could respond in an appropriate way to each change in its environment during the competition: 'CRSs had to make strategic decisions throughout the game: Which binaries to patch? Which patches to deploy? Which teams to attack, and with which exploits? Where should limited resources be spent?' 10

\section{System-Operator Relationship}

The motives for developing autonomous systems vary widely. Often, they amount to a desire to exceed human capabilities in some way, such as to perform a task with greater speed, accuracy, precision or over a longer period (speed being a particular concern in cyber contexts, where response times might often need to be on the order of milliseconds). The goal may alternatively be to remove humans from dangerous situations or hostile environments. Whatever the specific operational concern, the underlying technical need is for a system that can manage its own operation in a relevant way, rather than rely on interaction with a human operator. This 'user-facing' aspect is perhaps the most significant characteristic of an artificial autonomous system for the purposes of a legal analysis. It is captured in some definitions proposed by technical writers. Lin, Bekey and Abney define autonomy as:

\footnotetext{
${ }^{8}$ Joey Rideout, 'Cyber Reasoning Systems: Automating Cyber Warfare' (Medium, 8 August 2016) <https://medium.com/@joey_rideout/cyber-reasoning-systems-automating-cyber-warfare3329f339edeb > accessed 20 January 2020.

9 Teresa Nicole Brooks, 'Survey of Automated Vulnerability Detection and Exploit Generation Techniques in Cyber Reasoning Systems' (2019) 857 Advances in Intelligent Systems \& Computing $1083,1083$.

${ }^{10}$ Thanassis Avgerinos and others, 'The Mayhem Cyber Reasoning System' (2018) 16(2) IEEE Security \& Privacy 52, 56.
} 
The capacity to operate in the real-world environment without any form of external control, once the machine is activated and at least in some areas of operation, for extended periods of time. ${ }^{11}$

For reasons explained below, the phrase 'without any form of external control' should be read carefully, in the sense of 'operator interaction'. A more succinct definition is provided by Goodrich and Schulz:

A system with a high level of autonomy is one that can be neglected for a long period of time without interaction. ${ }^{12}$

Both of these definitions make it clear that a capacity for autonomous operation is reflected in the relationship between an autonomous system and its operator. That relationship is not severed; it remains one in which the system's designer or operator exerts, or has exerted, a form of control over the system by defining the task to which it is set and the manner in which it is able to interact with its environment. According to the United States Department of Defense:

Autonomy is a capability (or a set of capabilities) that enables a particular action of a system to be automatic or, within programmed boundaries, 'self-governing.' ${ }^{13}$

Two phrases in that definition, 'particular action of a system' and 'within programmed boundaries', are important. That an artificial system is able to operate without human interaction does not mean there are no human-imposed restrictions on the system's behaviour. Artificial autonomy essentially describes an approach to exercising human control over a system in circumstances where direct or real-time human interaction is infeasible or undesirable. In a robotics context (although equally applicable to software-only systems):

Autonomous means having the power for self government. Autonomous controllers have the power and ability for self governance in the performance of control functions. They are composed of a collection of hardware and software, which can perform the necessary control functions, without external intervention, over extended time periods. ${ }^{14}$

\footnotetext{
${ }^{11}$ Patrick Lin, George Bekey, and Keith Abney, 'Autonomous Military Robotics: Risk, Ethics, and Design' (2008) US Department of Navy, Office of Naval Research Report No ADA534697, 4 <http://www.dtic.mil/cgi-bin/GetTRDoc?AD=ADA534697> accessed 20 January 2020.

${ }^{12}$ Michael A Goodrich and Alan C Schultz, 'Human-Robot Interaction: A Survey' (2007) 1 Foundations \& Trends in Human-Computer Interaction 203, 217.

${ }^{13}$ US Department of Defense, 'DoD Directive No. 3000.09: Autonomy in Weapon Systems' (21 November 2012) 1. (Note that this Directive provides that the policies it outlines do not apply to cyber systems. However, this chapter takes the position that the cited definition of the abstract notion of 'autonomy' is as applicable to pure software systems as it is to software-driven hardware).

${ }^{14}$ Panos J Antsaklis, Kevin M Passino and S J Wang, 'An Introduction to Autonomous Control Systems' (1991) 11(4) IEEE Control Systems 5 (emphasis in original).
} 
A software application, whether or not it exhibits any degree of autonomous behaviour, is a tool. It is an implement used by a person or group to accomplish some goal. Its operation must, therefore, be directed by the operator toward that goal. Although autonomous systems are often described as being able to operate 'without external control', as in some of the definitions given above, that can be misleading in a discussion of their legal status. A software application's lack of interaction with an operator while it is running does not mean that its behaviour has not been defined by a person. Rather, it means that the intended behaviour was defined in advance of the software's activation and is then enforced by the code itself.

The behaviour of an autonomous software entity is ultimately dependent upon actions of people in relevant positions, notably its designer and operator, due to the nature of computers and software. Autonomous software entities are essentially sets of human-written instructions executed by human-constructed computing devices, including ordinary general-purpose computers, control units governing industrial processes, networking equipment such as routers or switches, 'internet of things' (IoT) devices that one might not readily recognise as computers, and so on. Although they may be highly specialised in design and purpose, such devices are nevertheless forms of ordinary stored-program computers, the defining characteristic of which is that instructions entered by a human programmer are stored in the machine's memory and drawn upon to govern its operation. Barring a major technological shift, tomorrow's autonomous systems will employ essentially the same technology.

The fact that even very complex programs are just sets of pre-defined instructions is often obscured in discussions about sophisticated autonomous behaviour, and indeed it is not always apparent to an observer that a complex system operating without human interaction is merely executing instructions rather than behaving independently of human influence. This is at least partly attributable to the use of software instructions which define how the software should respond directly to changes in its environment rather than only to instructions from a human operator. For example, even systems with only very simple and limited capabilities are often driven by programs with instructions of the form

if <X happens> then <do action A> else <do action B>

If ' $\mathrm{X}$ ' is something other than an input directly from an operator then such instructions can create the impression that the system itself is 'choosing' between two alternative courses of action, when in fact the choice was made in advance by the person or organisation responsible for writing the program; the expression of that party's will was merely waiting within the system's memory for the previously determined trigger to be detected. For example, a hypothetical cyber weapon might be encoded with a description of a specific type of database 
system which contains sensitive information belonging to an adversary, along with instructions describing a process for seeking, identifying and damaging systems matching that description. If such a cyber weapon detects a candidate system while scanning a network, an instruction like

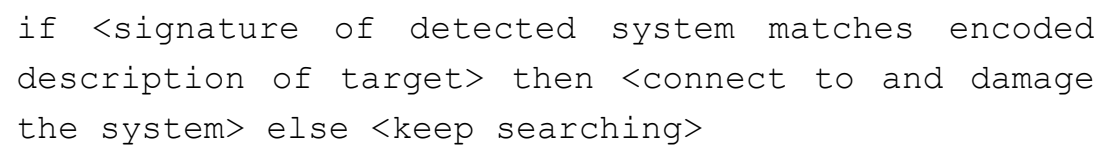

might create the appearance of the cyber weapon itself selecting targets, when actually the targets and the conditions under which they would be attacked were selected in advance by the system developers.

This reference to computing technology is included here because repeated references to autonomous systems having the capacity for 'choice' or 'truly autonomous' operation in the regulatory debate so far are potentially misleading. No computer is able to choose for itself whether or not to run a program stored in its memory, or to exercise discretion about whether or not to execute a particular instruction within a program; any such appearance of choice can only be the result of other instructions embedded in software. Fundamentally, the only function of a computer is to run whatever software is installed on it.

Autonomy, in a technical sense, is simply the ability of a system to behave in a desired manner, or achieve the goals previously imparted to it by its operator, without needing to receive the necessary instructions from outside itself on an ongoing basis. For simple tasks in well-understood environments, that might be achievable with a simple, static step-by-step set of instructions. Many firewalls fit this description. A firewall is a special-purpose computing device which is positioned at the edge of a network along the path taken by network traffic (data sent between computers) travelling into and/or out from that network. Its task is to examine each piece of traffic that attempts to pass through and decide, according to a set of programmed rules, whether that traffic is to be allowed through or blocked, in order to protect the network to which the firewall belongs. Firewalls are very common devices, being positioned at the edges of most corporate and government networks as well as being embedded within many consumer devices including personal computers and home internet routers. In the ordinary course of events, users are unlikely to need to interact with them, or even know they are present.

For more complex tasks, or tasks done in less predictable environments, autonomous operation might require that more advanced capabilities be encoded: to detect changes in the environment, to select a course of action from several possibilities in response to those changes, perhaps to recognise when a goal is not achievable, and so on. Some Intrusion Prevention Systems (IPS) might fall into this category. An IPS is a network security system which might incorporate several simpler sub-systems capable of performing a range of 
security-related functions along with some logic to control and co-ordinate those sub-systems according to the needs of the network's operator. For example, an IPS might include a firewall along with the ability to assess whether network activity might be malicious, reconfigure the firewall to block that activity, and perhaps repair damage caused by the malicious activity, such as by removing virus-infected email attachments or similar measures, ,all without requiring human intervention. Regardless of the complexity involved, though, autonomous software systems remain merely computer programs, written by human beings.

Understanding software autonomy as a form of control rather than as the absence of control is a necessary step toward identifying its legal implications. That it is a form of control is relatively easy to see when the behaviour of the system corresponds directly to software instructions entered by a human programmer. It is more difficult to see in the case of advanced software which, beyond simply operating without human intervention, may appear to exhibit some behaviour which has not been explicitly programmed by a person. Objections to development of highly autonomous military systems based on fears that they may select the wrong target or otherwise act in undesirable ways generally refer either explicitly or implicitly to this type of system.

Autonomous cyber weapons, like many other military systems for which autonomy is seen as an advantage, must be able to complete complex tasks in hostile and dynamic environments against adversaries who are able to learn and adapt. The exigencies of combat operations arguably make adaptability a more critical requirement for military systems than for those in civilian applications, as well as a greater challenge. The control functions of an autonomous software entity must be able to ensure the entity operates at a sufficiently high standard when there is a very high degree of uncertainty in the environment in which it operates. Behaviour of adversaries, active and passive defences, damage to systems and networks on which the entity operates and other events may all interfere with the operation of a cyber weapon such that some corrective action is needed outside of what might have previously been encountered in the course of the task being undertaken. In the case of an autonomous system, that corrective action must be initiated by the system itself rather than by a human operator. That is, when the system encounters a change in its environment such that the algorithm the system is using is no longer suitable, the system must be able to adapt that algorithm in order to achieve its goal. This type of capability can be found in some software systems in use today. For example, some radar systems offer 'constant false alarm rate' detection, wherein a radar system can adjust its own behaviour to compensate for varying levels of background noise and interference which might otherwise mask the presence of a target. Likewise, some computer worms and viruses employ 'polymorphic' code, or code which can rewrite itself without changing its core functionality, in order to evade security 
systems which might have been configured (or have adapted themselves) to detect the worm or virus in its previous form. Broadly, a software system which is able to alter its behaviour in response to changing circumstances is referred to as a 'self-adaptive system', or as software which employs an 'adaptive algorithm'. It is one source of the behaviours which define the software systems that are of most interest in the context of a discussion about the legal implications of software autonomy. Essentially,

Self-adaptive software evaluates its own behavior and changes behavior when the evaluation indicates that it is not accomplishing what the software is intended to do, or when better functionality or performance is possible. ${ }^{15}$

Importantly, such adaptation does not alter 'what the software is intended to do' (its purpose as defined by its human designers), although it may alter the low level steps that are taken in the course of fulfilling that purpose.

When utilising adaptive algorithms which enable a system to tune its own behaviour, the system may be operating as it was designed to even if the precise rules by which it is operating at a given time were not explicitly provided by a human operator (and may not even be precisely known to a human operator). Essentially, adaptive software employs higher level logic built into the software itself to generate whatever lower level operative rules are required as circumstances change. That higher level logic represents the operator's intent, and by altering its behaviour according to those higher level rules, the system is behaving in accordance with that intent.

Although adaptive techniques enable a system to alter its behaviour to an extent, their usefulness is limited by complexity. They rely on the system designer having a high degree of a priori knowledge about the system, its task and the environmental changes and disruptions that might be encountered, such that those factors can be mathematically modelled and represented in the software. In highly complex, poorly understood or unpredictable environments, or where the task to be completed is complicated, or even where the software itself is very complicated, it is not necessarily feasible to construct such a model in sufficient detail. In that case, another class of algorithm is likely to be employed.

Nonparametric algorithms, or those which do not rely on detailed mathematical models of the task or environment, are 'based on the use of more general models trained to replicate desired behaviour using statistical information from representative data sets. ${ }^{\prime}{ }^{16}$ That is, the software is provided with data representing situations that might be encountered in its intended operating

\footnotetext{
${ }^{15}$ Robert Laddaga, Paul Robertson, and Howie Shrobe, 'Introduction to Self-adaptive Software: Applications' in Robert Laddaga, Paul Robertson, and Howie Shrobe (eds), Self-adaptive Software: Applications (Springer 2003) 1.

${ }^{16}$ A Zaknich, Principles of Adaptive Filters and Self-learning Systems (Springer 2005) 3.
} 
environment, along with the desired responses to those stimuli, and it is 'trained' to generalise from the provided training data to arrive at an algorithm that will be effective in practice. This is a diverse field which draws on a range of techniques that enable software to operate in environments that are too complex or unpredictable, or about which too little is known, to be susceptible to the mathematical modelling required by traditional techniques. Generally, this 'intelligent' software works by emulating various aspects of biological cognitive processes, based on the premise that biological entities are often able to operate effectively with incomplete knowledge, in complex and ambiguous environments. ${ }^{17}$ The specific techniques employed are many, and the details are beyond the scope of this text; the most well-known techniques, which may be employed separately or in combination, are perhaps neural networks, fuzzy logic and genetic algorithms. The relevant advantage which all such techniques afford to the system designer is that they do not require detailed foreknowledge of all combinations of circumstances which the software entity may encounter once it is in operation. They allow the system designer to employ heuristics, approximation techniques and optimisation techniques to adapt the software's behaviour to circumstances which cannot be precisely foreseen.

A related issue is that of systems that continue to 'learn' after being put into operation. ${ }^{18}$ Learning, in this context, refers to the process of finding a generalised model which accounts for a set of observations, so that model can be applied when similar observations are made in the future. ${ }^{19}$ Rather than just responding to unexpected changes in its environment, a learning system is one that can improve its abilities over time by adjusting its 'rules' according to accumulated experiential knowledge; that is, allow information such as the performance of the system at previous tasks to be retained and used to tune behaviour in future tasks. Online learning (being learning that happens after a system is put into operation, as opposed to offline learning which happens during a development phase) is a considerably more ambitious control technique that is useful when the complexity or uncertainty of a situation prevents a priori specification of an optimal algorithm. It is another mechanism by which the rules by which a system operates at a given time may not be rules explicitly provided by a human operator. As with adaptive algorithms, though, generation of those rules according to the higher-level learning process is the behaviour intended by

\footnotetext{
${ }^{17}$ See, eg, Katalin M Hangos, Rozália Lakner and Miklós Gerzson, Intelligent Control Systems: An Introduction with Examples (Kluwer 2004) 1.

${ }^{18}$ D A Linkens and H O Nyongesa, 'Learning Systems in Intelligent Control: An Appraisal of Fuzzy, Neural and Genetic Algorithm Control Applications' (2002) 143(4) IEE Proceedings - Control Theory \& Applications 367.

${ }^{19}$ William Bialek, Ilya Nemenman and Naftali Tishby, 'Predictability, Complexity, and Learning' (2001) 13 Neural Computation 2409.
} 
the system's operator. The learning process which governs the overall behaviour of the software must be considered to represent the operator's intent.

'Intelligent' software generally applies techniques from the broader field of artificial intelligence (AI). ${ }^{20} \mathrm{AI}$ aims to understand the factors that make intelligence possible, and to employ that knowledge in creation of artificial systems that can operate in ways which, if observed in living beings, would be considered intelligent. That is, systems that can respond appropriately to changing circumstances and goals, take appropriate actions when provided with incomplete information and, if needed, 'learn' from experience.

Despite the complexity of software which relies on these advanced algorithms, it is not fundamentally distinct from simpler automated or manual processes. They are all still means of achieving some human-defined goal. Regardless of its complexity, autonomous software amounts to a set of instructions guiding a system toward such a goal. Those instructions may endow a system with a capacity for complex actions and responses, including the ability to operate effectively in response to new information encountered during operations which may not be precisely foreseeable to a human operator. However, that does not constitute independence from human control in any sense. Rather, it is best seen as control applied in a different way, in advance rather than in real time.

\section{Autonomy as a Property of a System}

Autonomy is a property of a technological system which may be realised by diverse means. It does not connote the presence of a specific technology nor a particular type of device nor a certain behaviour. It is, fundamentally, the ability of a system to perform the task assigned to it, whatever that may be, with less interaction with a human operator than a manual system would require. Implicitly, that is achieved by enabling the system to interact directly with its environment rather than have it refer every decision to a human. For the purposes of investigations in non-technical fields, software autonomy primarily affects the relationship between the system and its human operator, not the nature of the system's task nor the precise manner in which it performs that task. Two points in particular are relevant in an investigation of the legal status of autonomous systems.

First, autonomous systems will perform their assigned tasks in place of humanoperated manual systems, but the outcomes will not necessarily differ from those which would have been achieved had the tasks been done manually by humans. There is nothing in the concept of software autonomy that supports an inference that an autonomous system must necessarily perform a task in a different

\footnotetext{
${ }^{20}$ See generally Stuart Russell and Peter Norvig, Artificial Intelligence: A Modern Approach (3rd edn, Prentice Hall 2009).
} 
manner than would a human or team of humans performing the same task manually. Of course, one of the motivations for developing increasingly autonomous systems is to achieve superior outcomes. The persistence of systems that do not require constant human interaction; the ability to quickly integrate data from many sources; the capacity for greater speed, accuracy or precision; and the ability to take greater risk than could be taken via a manual approach; among other benefits of autonomous systems, will certainly aid both military and other operations. However, such differences, while very important operationally, are somewhat peripheral to the legal aspects of autonomy. Remotely piloted aircraft (RPA), for example, already allow for a high level of persistence without necessarily exhibiting any capabilities associated with a high level of autonomy and without raising the same legal questions. In assessing the legal implications of a particular development path, or a particular set of technologies, the focus must be kept on the capability of interest rather than on other capabilities that may be present in the same system. In the case of autonomous software, it is not useful to attempt to attribute specific behaviours to an application merely on the basis of it being described as having some capacity for autonomous operation; all that one can reliably say on that basis is that the human operator's direct involvement in part or all of the software's performance of its assigned task will be reduced or removed. The mere fact of reassigning a task from a human to a computer does not necessarily alter the performance of that task.

Second, it is incorrect to describe autonomous systems as being 'independent' ${ }^{21}$ systems that operate 'without human control'.22 The relationship between human and software is not severed, it is only modified. Choices made by developers in the design stage will shape the behaviour of the systems they create from then on, for both technical and operational reasons. On a technical level, as explained above, everything that an autonomous system does (barring malfunctions and interference) is ultimately the result of executing a set of software instructions written by human developers. On an operational level, it is an obvious practical necessity that an autonomous system be constrained to behave consistently with its purpose. In a military context, a cyber weapon is only one component in a State's armed forces. Its use must be in accordance with higher level plans and established procedures as well as with the capabilities and practices of other units and support structures, and the autonomous system's role would often be only one component in a larger coordinated effort. Mission planners and commanders will set goals and impose constraints which must be satisfied, and significant human effort might be expended gathering intelligence and otherwise facilitating the operation. For example, the well-known Stuxnet

\footnotetext{
${ }^{21}$ Markus Wagner, 'Taking Humans Out of the Loop: Implications for International Humanitarian Law' (2011) 21(2) Journal of Law, Information \& Science 155, 159.

${ }^{22}$ Gary E Marchant and others, 'International Governance of Autonomous Military Robots' (2011) 12 Columbia Science \& Technology Law Review 272, 273.
} 
worm, which was used to disable centrifuges at the Natanz nuclear facility in Iran, has been described as possessing a degree of autonomous capability:

Considering that there was very good chance that no Internet connectivity would be available (only access to the internal network), Stuxnet developers put all of its logic in the code without the need of any external communication. As such the Stuxnet was an autonomous goal-oriented intelligent piece of software capable of spreading, communicating, targeting and self-updating; $\ldots{ }^{23}$

Although much is unknown about the use of Stuxnet, security researchers from Symantec have outlined a possible attack scenario based on their analysis of the worm's capabilities, which demonstrates a plausible degree of human involvement:

First, the attackers needed to conduct reconnaissance. As each PLC [programmable logic controller; the device targeted by Stuxnet] is configured in a unique manner, the attackers would first need the ICS's [industrial control system; the network environment in which the PLCs exist] schematics. These design documents may have been stolen by an insider or even retrieved by an early version of Stuxnet or other malicious binary. Once attackers had the design documents and potential knowledge of the computing environment in the facility, they would develop the latest version of Stuxnet...Attackers would need to setup a mirrored environment that would include the necessary ICS hardware, such as PLCs, modules, and peripherals in order to test their code. The full cycle may have taken six months and five to ten core developers not counting numerous other individuals, such as quality assurance and management. In addition their malicious binaries contained driver files that needed to be digitally signed to avoid suspicion. The attackers compromised two digital certificates to achieve this task. The attackers would have needed to obtain the digital certificates from someone who may have physically entered the premises of the two companies and stole them, as the two companies are in close physical proximity. To infect their target, Stuxnet would need to be introduced into the target environment. This may have occurred by infecting a willing or unknowing third party, such as a contractor who perhaps had access to the facility, or an insider. ${ }^{24}$

\footnotetext{
${ }^{23}$ Stamatis Karnouskos, 'Stuxnet Worm Impact on Industrial Cyber-Physical System Security' (Paper presented at IECON 2011 - 37th Annual Conference of the IEEE Industrial Electronics Society, Melbourne, Victoria, Australia, 7-10 November 2011) 4492.

${ }^{24}$ Nicolas Falliere, Liam O Murchu and Eric Chien, 'W32.Stuxnet Dossier' (v1.4, Symantec Security Response, February 2011) 3

<https://www.symantec.com/content/en/us/enterprise/media/security_response/whitepapers/w32 _stuxnet_dossier.pdf $>$ accessed 20 January 2020.
} 
In another, albeit robotics-related, example, Boothby describes the level of human involvement that would be required in conducting an attack with an autonomous aircraft: ${ }^{25}$

\begin{abstract}
A flight plan will have been prepared and filed by a person who will decide on the geographical area that is to be searched, the time period within which the search may take place, the areas where the aircraft may loiter and for how long, and that person will programme these important requirements into the flight control software. The platform will be fuelled by a person thus defining the maximum endurance of the mission. Operational planners will decide what weapons will be carried and how they are to be fused, and stores will be loaded by people before takeoff. The sensors on which the autonomous aspect of the mission depends will have been designed and built by people and will be controlled by similarly designed software. Those designers and/or the mission planners will have prescribed the level of mechanical recognition that is to be achieved before an object is recognised as a target and, thus, before an attack will be undertaken.
\end{abstract}

It may be assumed that analogous tasks would be performed by humans in relation to other operations involving autonomous software. In these ways a human hand always provides some degree of guidance despite a possible lack of direct supervision. The Defense Science Board of the United States Department of Defense (writing about autonomous vehicles) expresses the dependence of autonomous systems on humans more generally:

It should be made clear that all autonomous systems are supervised by human operators at some level, and autonomous systems' software embodies the designed limits on the actions and decisions delegated to the computer. Instead of viewing autonomy as an intrinsic property of an unmanned vehicle in isolation, the design and operation of autonomous systems needs to be considered in terms of human-system collaboration. ${ }^{26}$

\title{
Human-System Collaboration
}

Despite that, on the technical level, autonomous capabilities are inherently a matter of degree, it is common in the non-technical literature on autonomous systems to attempt to categorise particular systems according to one taxonomy or another. One popular classification scheme, often cited in discussions about autonomous weapon systems, distinguishes between 'automatic', 'automated',

\footnotetext{
${ }^{25}$ William Boothby, 'How Far Will the Law Allow Unmanned Targeting to Go?' in Dan Saxon (ed), International Humanitarian Law and the Changing Technology of War (Martinus Nijhoff 2013) 56.

${ }^{26}$ Defense Science Board, 'The Role of Autonomy in DoD Systems' (July 2012) US Department of Defense Task Force Report, 1-2.
} 
and 'autonomous' systems. These terms have been used somewhat differently by different authors, but the essential distinctions are as follows: ${ }^{27}$

- 'Automatic' refers to very simple devices which perform well-defined tasks and may have the ability to respond in pre-set ways to external stimuli. Systems which can operate unattended but which have little or no ability to receive and act on feedback from their environment are sometimes described as 'automatic'.

- 'Automated' may be used synonymously with 'automatic' but may also refer to systems which follow more complex sets of rules in normal operation and in responding to disturbances, such that they can perform more complex tasks or operate in more complex environments. Examples include automated telephone support lines that can respond in limited ways to various queries, or some existing weapon systems.

- The varying uses of the term 'autonomous' among authors reflects the uncertainty that surrounds the nature of these new technologies. The general view is that autonomous systems go beyond automated systems in some way, but the precise criteria vary. Some authors describe systems as 'autonomous' when they exhibit some ability to adapt their own behaviour in response to changing circumstances. ${ }^{28}$ Others use the term to indicate that some threshold level of complexity in the system, its task or its operating environment has been reached. ${ }^{29}$ Still others say autonomous systems are those with some degree of 'independence' from their human operators. ${ }^{30} \mathrm{~A}$ further subdivision within this category is between 'semi-autonomous' and 'fully autonomous' systems. The claimed difference is that fully autonomous systems are those which are designed to operate entirely without human involvement once activated while semi-autonomous systems would require some form of active human involvement in relation to some or all functions. A range of views have been expressed over whether fully autonomous systems would entirely disallow human involvement, or simply not require it, and about the extent of human involvement required in semi-autonomous systems.

\footnotetext{
${ }^{27}$ Paul Scharre, 'Autonomous Weapons and Operational Risk' (February 2016) Center for a New American Security, Ethical Autonomy Project, 12

<https://www.cnas.org/publications/reports/autonomous-weapons-and-operational-risk> accessed 20 January 2020.

${ }^{28}$ Kenneth Anderson and Matthew C Waxman, 'Law and Ethics for Autonomous Weapon Systems: Why a Ban Won't Work and How the Laws of War Can' (Hoover Institution, 9 April 2013) 6.

${ }^{29}$ Rebecca Crootof, 'The Killer Robots Are Here: Legal and Policy Implications' (2015) 36 Cardozo Law Review 1837, 1854 .

${ }^{30}$ Chantal Grut, 'The Challenge of Autonomous Lethal Robotics to International Humanitarian Law' (2013) 18(1) Journal of Conflict \& Security Law 5.
} 
Some commentators create finer distinctions within each of those levels depending on the degree of necessity of the human operator's contribution, the realistic possibility for successful human intervention once the system is deployed, and so forth.

Attempts to define taxonomies of autonomy are further complicated by differing views on whether categories should be based on the degree and type of human interaction with the system, or the complexity of the system and its behaviour. The two variables are both plausible bases for categorisation (if one believes that categorisation is appropriate), but each captures only one aspect of autonomy, and any simple discrete taxonomy fails to reflect the fact that the levels of autonomy exhibited by existing and proposed systems may be expected to vary in complex ways. Capacities for autonomous operation vary widely, as do the ways in which tasks are allocated between an operator and an autonomous system, and the behaviour of a system may be expected to change according to both the specific task being performed and the current state of the environment in which the system is operating. Establishing the relative degrees of control exercised by a human operator and a software system in respect of a particular action for legal or other purposes may be a complex process. The Defense Science Board offers this view of the variability of degrees of autonomy from a cognitive science perspective:

Cognitively, system autonomy is a continuum from complete human control of all decisions to situations where many functions are delegated to the computer with only high-level supervision and/or oversight from its operator. Multiple concurrent functions may be needed to evince a desired capability, and subsets of functions may require a human in the loop, while other functions can be delegated at the same time. Thus, at any stage of a mission, it is possible for a system to be in more than one discrete level simultaneously. ${ }^{31}$

The complexity of defining a degree of system autonomy is well demonstrated by consideration of the various dimensions along which autonomous behaviour may vary.

Rather than simply requiring more or less guidance from a human operator, a software entity may require guidance in different forms: ${ }^{32}$ the software might determine available options in some situation and rely on a human to select one; the software might recommend a particular option or not; it might begin to

\footnotetext{
${ }^{31}$ Defense Science Board (n 26) 4 .

32 See, eg, Thomas B Sheridan and William L Verplank, 'Human and Computer Control of Undersea Teleoperators' (Technical Report, Massachusetts Institute of Technology, 14 July 1978) 8-17; NIST Engineering Laboratory, 'Autonomy Levels for Unmanned Systems' (National Institute of Standards and Technology, 6 June 2010) < http://www.nist.gov/el/isd/ks/autonomy_levels.cfm> accessed 20 January 2020.
} 
undertake a course of action and give an operator a chance to override that choice; it might complete the whole task and report back (or not) afterwards.

A human may be required to play the role of a hands-on 'operator' in some cases and a hands-off 'supervisor' in others. They may alternatively be more of a 'collaborator', sharing tasks with an autonomous entity, with the allocation of specific tasks being negotiated between them, or perhaps controlled by a third party. ${ }^{33}$ In a collaborative scenario, either the software or the human might have direct control of a specific task at a specific time with the other party assisting, or they might be working independently.

Just as the activities of an autonomous software entity would generally form one part of a larger coordinated operation, autonomous capabilities are likely to exist in specific sub-systems performing specific functions rather than be applied to the system as a whole. A cyber weapon might be trusted to locate and identify potential targets autonomously, but be required to seek human confirmation before attacking them. The level of autonomy displayed by an entity might therefore vary according to the specific task being performed during an operation, ${ }^{34}$ enlivening the possibility that a system may be operating at more than one 'level' of autonomy simultaneously, with respect to different tasks.

Finally, the level of autonomy exhibited by a system might vary with circumstances that arise during an operation. A system that can operate entirely unassisted in normal circumstances might refer a decision to an operator if an unexpected problem arises, or if an unanticipated opportunity presents itself.

The US Department of Defense summarises the variability inherent in autonomous software operation: 'The key point is that humans and computer agents will interchange initiative and roles across mission phases and echelons to adapt to new events, disruptions and opportunities as situations evolve.' 35

\section{Conclusion}

Autonomous software entities, whatever advanced capabilities they possess, remain exactly that: software. They are tools wielded by human operators, sequences of human-written instructions executed by human-constructed computers for human-defined purposes, qualitatively identical to any other

\footnotetext{
${ }^{3}$ See, eg, Jean Scholtz, 'Theory and Evaluation of Human Robot Interactions' (Paper presented at 36th Annual Hawaii International Conference on System Sciences, Hawaii, 6-9 January 2003); Marti A Hearst, 'Trends \& Controversies: Mixed-Initiative Interaction' (1999) 14(5) IEEE Intelligent Systems 14 .

34 This phenomenon is emerging in some current weapon systems: Rain Liivoja, Kobi Leins and Tim McCormack, 'Emerging Technologies of Warfare' in Rain Liivoja and Tim McCormack (eds), Routledge Handbook of the Law of Armed Conflict (Routledge 2016), ch 35, s 2.1.

35 Defense Science Board (n 26) 27.
} 
software. They will generate the rules by which they operate if that is what they are programmed to do, but in doing so, their ties to their developers are not severed; the process of generating those rules in pursuit of their designed purpose is the behaviour that their developers intended. Despite that the core meaning of autonomy is 'self-governance', software cannot be regarded as a root cause of its own behaviour, at least for legal purposes, in the same sense that a human can (leaving aside the question of whether and to what extent human beings truly determine their own behaviour).

The most important point to take away from this chapter is that 'autonomy', as the concept applies to software, does not mean freedom from of human control; it is, rather, a form of control. For obvious practical reasons, autonomous software entities must be directed toward fulfilling the purpose for which they were designed. Autonomous control is the means by which that is achieved. If control can be conceived of generally as the set of measures taken to determine the behaviour of a software entity, then specific control actions can be applied by a human operator either in advance of an operation, in the form of programmed behaviours (whether proactive behaviours or responses to environmental stimuli), or during an operation, in response to some indication that a control action is required (such as how an RPA pilot guides the aircraft in response to imagery captured by the RPA's camera). Autonomous control is a control paradigm which relies on control inputs applied to a system in advance, to the partial or complete exclusion of human interaction applied during an operation, so as to realise some practical benefit of relevance to a mission (speed, accuracy, persistence, stealth, and so on).

Autonomy is inherently a matter of degree. Practical limitations on a system's ability to define its own behaviour will always exist, whether as 'hard' limits in the form of programmed behaviours or 'soft' limits in the form of environmental constraints which the system is unable to overcome. The objective to be achieved in a specific mission and the need to interoperate with other entities, whether human or artificial, in pursuit of that objective, must necessarily constrain 'selfgovernance' to some extent. Nor will a system's degree of autonomy necessarily be constant; in practice it may be expected to vary with respect to the specific function in question and the circumstances in which the system is operating.

For the purposes of a legal analysis, that means that attempts to classify software systems as autonomous or not in a binary sense are highly error-prone. If autonomous capability per se is to be used as the basis of a legal argument, significant care must be taken to select a representation which is technically accurate as well as legally relevant. Alternatively, perhaps the challenge for lawyers studying software autonomy is to relate the human and software behaviours that are the signature of autonomous operations directly to those that are subject to legal regulation. 